\title{
LA CONSTRUCTION DES IDENTITÉS COMMUNICATIONNELLES COMME VOLONTÉ DE FAIRE MONDE COMMUN. EXEMPLE DES INTERACTIONS CONFLICTUELLES DANS L'ÉMISSION ON N'EST PAS COUCHÉ
}

\author{
Gwenolé Fortin ${ }^{1}$
}

S'appuyant sur un corpus constitué de séquences issues de l'émission de France 2 On n'est pas couché, il s'agit de décrire les interactions langagières - argumentatives - comme élément constitutif à la fois de l'identité sociale des interlocuteurs et en même temps constituées par les sujets en interaction.

Fondé sur les travaux inspirés par la sociolinguistique interactionnelle et l'interactionnisme symbolique, il s'agit d'adopter une démarche analytique considérant «l'affrontement» verbal comme un fait de surface (qui apparaît dans la mise en mots et la mise en scène des interactions). En dépassant le paradigme classique des travaux sur l'argumentation (paradigme persuasif), il s'agit de montrer comment ces interactions conflictuelles procèdent bien davantage d'une dynamique de négociation identitaire et d'un processus de co-construction de sens.

1 Gwenolé Fortin est Docteur en sciences du langage et maître de conférences en sciences de la communication à l'IUT de Saint-Nazaire (Université de Nantes), chercheur au laboratoire LEMNA (Université de Nantes).

Recherches en communication, $\mathrm{n}^{\circ} 42$ (2016). 


\section{Introduction}

L'article s'intéresse ici aux stratégies de construction (ou plutôt de co-construction) des identités mobilisées par les «interactants » lorsqu'ils se trouvent (et s'installent même) dans des situations discursives polémiques (Declercq, 2003). Pour ce faire, nourri du cadre théorique propre à E. Goffman - l'interactionnisme symbolique l'article s'appuie sur un corpus d'extraits de l'émission On n'est pas couché, diffusée depuis 2006 sur France 2.

Nous partons d'un constat : les débats médiatiques, en particulier dans cette émission, se caractérisent par un ensemble de pratiques et d'occurrences conflictuelles. Ils se définiraient même d'emblée par cette dimension polémique (Rouquette, 2002) et ces joutes verbales dont parlait déjà le psycholinguiste Uli Windisch dans le K.O verbal (Windisch, 1986) ; ce que confirment par ailleurs les commentaires en chaine que suscitent ces séquences reprises sur les forums de Youtube, Dailymotion ou Facebook. L'affrontement des sujets, l'adversité et le conflit, semble être l'une de leurs caractéristiques principales, et le lien structurel entre tous ces débats pourrait même se résumer en un mot : le «clash» (ce mot apparaissant dans quasiment chacun des liens relayant ces séquences - cf note précédente) ; terme employé également par les interlocuteurs eux-mêmes pour qualifier le débat:

Christophe Hondelatte (à Laurent Ruquier) : c'est bon vous l'avez votre clash $[\ldots]$

De fait, ces débats s'inscrivent dans une dynamique dialogique d'affrontement, relation duale qui leur tient lieu de définition même : nous aurions ainsi affaire à une sorte « d'objet creux », « vide de sens », ou immobile pour reprendre l'expression de Marianne Doury (Doury, 1997).

\subsection{Un nouveau paradigme : la co-construction d'un monde commun}

C'est effectivement l'un des points de vue que l'on peut adopter sur ces débats. Mais ce n'est pas le seul. Il est ainsi possible de (re) considérer « l'affrontement » comme un fait de surface - qui apparait certes dans la mise en mots, et en scène du débat (avec l'utilisation de split screen) - mais qui procède aussi d'un processus de co-construction de sens tout autant qu'un processus de co-construction du réel, c'està-dire une volonté communicationnelle de faire monde commun. Les interactions conflictuelles seraient davantage l'expression d'une relation interpersonnelle à travers laquelle les interlocuteurs, dans une 
dynamique interlocutoire conflictuelle mais aux règles partagées, coconstruisent un univers référentiel commun; c'est-à-dire co-construisent un monde qui se dit, dont la «communauté »- au sens de ce qui est commun - se construit par le langage : le langage envisagé ici comme instrument de l'invention/négociation du monde (Fortin, 2015).

Il ne s'agit donc pas de nier la dynamique conflictuelle caractérisant ces débats mais plutôt d'adopter une autre perspective : reconnaître la dimension agonistique des débats - comme élément constituant de leur contrat de communication télévisuel (Charaudeau, 1991) - mais voir aussi comment cela produit du sens. Dans cette perspective, ce qui se joue alors entre les débattants, bien davantage qu'un conflit, c'est une coopération communicative très forte - à l'intérieur d'un genre discursif qui est posé, et dont ils connaissent les règles et les contraintes spécifiques ; règles et contraintes qu'ils construisent en les reproduisant, de telle sorte qu'ils produisent un sens. Ces interactions conflictuelles visent ainsi à « faire faire », « faire croire » ou " penser », c'est-à-dire faire adhérer à une réalité (sociale, politique ou culturelle) qui produit des effets.

\subsection{Notre Problématique}

Notre problématique est ainsi la suivante : ces débats - en tant qu'activité communicationnelle, réglée et porteuse d'enjeux identitaires, signifient la co-construction d'un monde commun; comme l'écrit John Gumperz, « interagir c'est aussi partager quelque chose » (Gumperz, 1989, 155). Ces débats - s'articulant autour de déclinaisons identitaires - doivent alors être pensé comme un espace contractuel de genèse intersubjective à travers laquelle les sujets actualisent un rapport commun au monde ; de sorte que le monde n'est pas déjà là, naturel (vs social ou symbolique), mais qu'il ad-vient : autrement dit qu'il est à co-construire, c'est-à-dire à négocier en permanence par le biais du langage - y compris donc dans le conflit. Il s'agit ainsi d'illustrer l'interactionnisme symbolique au moyen d'un exemple privilégié : une émission polémique qui produit du sens.

\section{Propriétés de l'objet d'étude}

Notre corpus appartient à la catégorie des corpus médiatiques, en l'occurrence télévisuels. Dans ce cadre, la situation de communication relève d'un dispositif spécifique - triangulaire ou tripolaire - avec une interaction symétrique entre les interlocuteurs directs et une 
communication (car il s'agit bien d'une communication) asymétrique entre les interlocuteurs d'une part, et les récepteurs, témoins muets de l'échange d'autre part (téléspectateurs ou spectateurs).

Cette situation communicationnelle particulière, télévisuelle, est gérée par un contrat médiatique constituant - selon la terminologie de Nel (Nel, 1990) - le niveau de "l'espace télévisé »; l'autre niveau ou instance - liée à l'interaction, au sein de ce contrat médiatique, est constitué par le réalisateur : il s'agit ici du « débat télévisable».

\subsection{L'émission On n'est pas couché}

L'émission On n'est pas couché se donne ouvertement pour objectif de présenter deux interlocuteurs qui se font face : un chroniqueur - en position de critique -, et un invité, venu faire la promotion de son travail. L'émission est emblématique du genre " débat télévisé » (Nel, 1990) par sa disposition scénique (circulaire), le choix des invités (écrivains, chanteurs ou comédiens populaires), le statut de l'animateur (Laurent Ruquier) et la notoriété de ses chroniqueurs vedettes : Eric Naulleau, Eric Zemmour, Natacha Polony, Audrey Pulvar puis Aymeric Caron et Léa Salamé. C'est aussi la principale émission de talk-show - sans réelle concurrence sur une chaîne publique - présentant chaque samedi soir des débats (souvent conflictuels) à une heure de grande écoute (22h50).

Cela étant, parler des débats ne signifie pas qu'ils soient tous homogènes (ils ne se situent pas tous sur le même plan et ne produisent pas les mêmes effets) mais ils partagent une caractéristique commune : leur inscription dans une dynamique conflictuelle (mise en relief par la réalisation avec l'utilisation de split screen). Ces débats issus de l'émission de France 2, souvent très houleux, produisent des discours qui dans leur manifestation textuelle sont indissociables de leurs conditions de production, autant que des dispositifs scéniques dont ils sont l'une des composantes intrinsèques, l'émission dans laquelle ils s'inscrivent reposant toujours sur les mêmes principes et dispositifs techniques, avec la même chronologie, les mêmes rubriques, les mêmes chroniqueurs, etc. et donc aussi les mêmes attentes de la part des spectateurs et téléspectateurs. Les différents protagonistes " obéissent " ainsi à un certain rituel communicationnel. Ce modèle communicationnel (au sens large, c'està-dire englobant des postures et des motivations particulières) est présent-même inconsciemment - chez les invités et leurs interlocuteurs (animateur et chroniqueurs); et selon les analogies de la situation de communication avec le modèle ainsi constitué, les interactants auront 
plus ou moins tendance à se conformer aux normes de communication dont est porteur le modèle ; et se feront par ailleurs reconnaître au travers de celui-ci. Ce qui se joue alors dans l'interaction c'est une confirmation de la relation et de l'appartenance à une même culture unissant les différents acteurs, invités et chroniqueurs (y compris dans des interactions à caractère conflictuel) : « Nous avons "performé" les codes de la culture à laquelle nous appartenons, et cette coperformance nous a rassurés quant à notre qualité de membres [...] » (Winkin, 2001, 14).

\section{Cadre théorique}

\subsection{Le Grand Partage platonicien}

La tradition épistémologique classique (platonicienne) a radicalement conditionné notre rapport au monde. Dans le système platonicien, le logos s'oppose à la doxa, qui est chargée de tous les maux : influençable, irrationnelle, émotionnelle, etc. Dans le paradigme platonicien, le monde est ainsi partagé en deux sphères hétérogènes : d'un côté le monde naturel (de la Raison), le monde des faits ou des choses telles qu'elles sont (logos) -, de 1'autre le monde social (passionné ; et « à dé-passionner »), le monde du Sujet, des représentations et des conflits qui vont avec $($ doxa $)$.

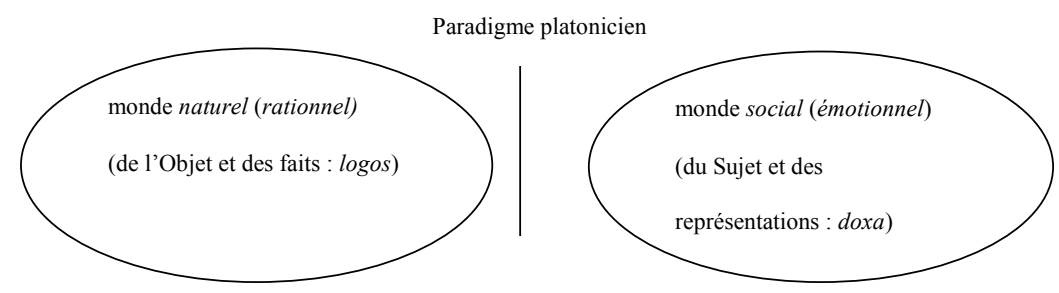

Figure 1

Dans la lignée du système platonicien, pour la linguistique informationnelle (relevant d'une linguistique immanentiste héritée de la philosophie platonicienne du langage), le langage dit le monde, il ne le crée pas - ou alors seulement sur le plan symbolique. La linguistique est ainsi fondée sur la «métaphore du conduit » (communication codique : schéma Emetteur-Récepteur) et le langage est fondamentalement informationnel (vs monde naturel), c'est-à-dire qu'il transporte l'information de ce sur quoi il porte et qu'il dédouble. 


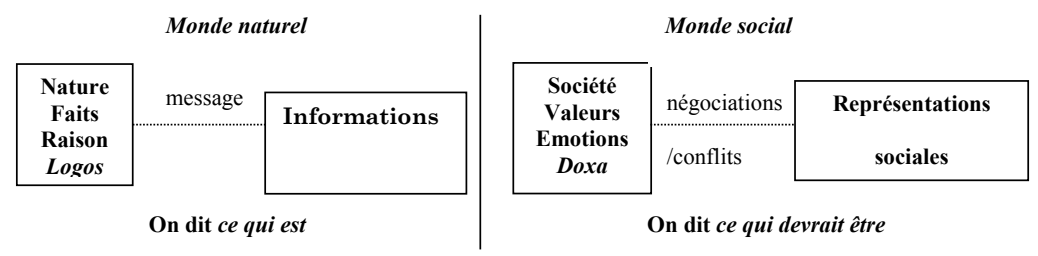

Figure 2

Aussi, le paradigme classique des travaux sur l'argumentation, construit dans la perspective logocentrique de la néo-rhétorique platonicienne, inscrit les discours argumentatifs dans une visée persuasive : il s'agirait pour les locuteurs de viser à convaincre leurs interlocuteurs (Fortin, 2006).

Notre approche est autre : les débattants, sur les plateaux de télévision - en particulier dans l'émission On n'est pas couché - visent non pas à convaincre leurs interlocuteurs mais bien davantage à se conformer à ce que le public (spectateurs et téléspectateurs) attend d'eux. L'argumentation doit alors être pensée comme un type particulier de discours consacré à un souci de présentation de soi $\left(\right.$ ethos $\left.^{1}\right)$ : les débattants - y compris dans l'interaction conflictuelle - cherchent à construire, défendre ou imposer une image d'eux-mêmes qui fasse sens pour les téléspectateurs, et qui fonctionne comme une sorte d'horizon d'attente, de mise en perspective de ce qu'ils sont censés être ou ne pas être. L'enjeu est de co-construire leur identité et donc de « dire »qui ils sont.

Mickaël Youn : je sais pas ce que vous faites ...

Eric Zemmour : moi je suis journaliste, je suis journaliste...

Mickaël Youn : vous bossez où ?

Eric Zemmour : je suis journaliste au Figaro, je suis journaliste politique depuis 20 ans et voilà

Mickaël Youn : qu'est-ce que vous faites là ? vous faites tout et n'importe quoi $[\ldots]$

Eric Zemmour : si ya que vous qu'avez le droit de passer à la télévision faut l'dire [...]

1 Cette notion apparait dans les sciences du langage avec Oswald Ducrot, dans le cadre d'une pragmatique sémantique. Voir les travaux de Ruth Amossy $(1999,2014)$. 
Eric Naulleau : à chaque fois c'est la même chose : vous êtes qui ? vous êtes qui ? ${ }^{1}$

Leur confrontation fait sens dans le souci réciproque de maximiser la cohérence de leurs identités respectives. C'est autour de cet enjeu, identitaire, que se construisent alors des éléments d'argumentation : le débat permettant, et sollicitant, l'expression d'identités situationnelles concurrentes à travers un effort commun de mobilisation de/des l'interlocuteur(s).

Il s'agit donc de questionner la communication polémique dans ce cadre médiatique - non plus selon l'héritage platonicien de la philosophie du langage (modèle codique de la communication), mais selon une logique identitaire, portant un regard sur les interactions comme signifiant autant par leur existence même que par leur contenu propositionnel. Le but est alors de détailler la logique identitaire inscrite dans ces interactions et de montrer que le sens de ce qui est dit dépasse, et de beaucoup, le strict contenu propositionnel ; lequel, en l'occurrence, s'avère souvent bien modeste. Une telle approche théorique s'articule ainsi autour du discours argumentatif envisagé comme moteur dans la construction et la négociation des réalités sociales, c'est-à-dire que l'on postule que celles-ci « émergent des activités de communication [...] qui contraignent l'organisation et la signification des discours dans le même temps que les discours produits dans des contextes similaires façonnent les propriétés de ces activités »(Burger, 1994, 251). En somme, il s'agit pour les interactants s'affrontant de la sorte de se définir et de se positionner à l'égard du contradicteur en présence, en d'autres termes de se dire eux-mêmes en s'opposant (Amossy, 2014). C'est donc dans une perspective « constructionniste » de l'identité, renvoyant à la notion goffmanienne de « soi » (self), que nous nous intéressons ici aux aspects discursifs des présentations de soi dans des situations d'interactions conflictuelles.

\subsection{L'interactionnisme symbolique}

Cette perspective de recherche, envisageant l'identité comme l'un des plans d'organisation du discours argumentatif, se doit d'intégrer la notion de rapport de places et s'inscrire dans le prolongement

1 Pour ce qui relève des conventions de transcription (volontairement simplifiées) : ... (superposition des voix), [...] (ellipse), MAJUSCULE (le locuteur crie). 
épistémologique de l'interactionnisme symbolique, développé en partie par les travaux d'Erving Goffman. Les débattants - loin de se contenter " de transmettre de l'information » et de chercher à se convaincre jouent avec l'identité de leurs interlocuteurs en leur assignant différentes places. ${ }^{1}$ Il s'agit donc de penser l'identité comme le produit intériorisé des interactions vécues par un sujet, chaque locuteur se définissant par une sorte de " profil identitaire » construit dans la dialectique d'une reconnaissance intersubjective ; ce profil identitaire étant à la fois le motif inaugural et le produit de l'interaction verbale. L'interactionnisme symbolique de Goffman, qui relève d'une approche dramaturgique des interactions sociales, accorde une place tout à fait centrale à la définition que se font les protagonistes de la situation d'énonciation, celle-ci relevant d'une co-construction et n'étant plus une donne statique - en rupture donc avec le paradigme traditionnel « locuteurauditeur » envisagé par le modèle du code et relevant d'une linguistique immanentiste héritée des premières philosophies du langage.

Centrée sur la notion d'identité ${ }^{2}$, cette perspective s'écarte ainsi des conceptions conventionnelles (ou classiques) de l'argumentation qui « posent comme objectif un discours argumentatif idéal, qui serait avant tout rationnel, libéré des contraintes de temps, obéissant aux règles du dialogue raisonné à la Habermas ou à la Grootendorst \& van Eemeren » (Doury et al., 1998, 9). Ici, le caractère argumentatif des discours ne dépend pas d'un jugement de rationalité mais de caractéristiques intrinsèques au discours, en particulier de la relation qu'il construit à un contre-discours qu'il met en scène et par rapport auquel il se définit. L'activité d'argumentation ne passe donc plus par l'évaluation des figures argumentatives « classiques » (paradigme de la persuasion) mais relève de la négociation, de la co-construction des identités.

S'écartant des outils élaborés par les théories rhétoriques et argumentatives, inscrites dans la perspective logiciste de l'argumentation - conception essentiellement unilatérale de la communication -, il s'agit d'envisager, avec Plantin par exemple, que « l'argumentation

1 La notion de place doit être distinguée ici de celle de rôle et de statut (utilisée dans les sciences sociales) : la place n'est pas le rôle ou le statut mais l'identité que s'assigne le locuteur; elle est fonction du contexte de la situation de communication, fonction des interlocuteurs, des intentions de communication et de la représentation des enjeux de l'échange.

2 Paradoxalement, il s'agit là d'une perspective peu étudiée, en dépit d'une utilisation notionnelle qui est, elle, très répandue. 
est dialectique ; son langage n'est pas un langage d'objets, mais un langage habité par les interlocuteurs et marqué par leurs points de vue. » (Plantin, 1990, 232). L'identité, et le social qui la constitue, sont alors rendus « accessibles » par les formes et les activités discursives des interlocuteurs. Aussi, au-delà de la prise en compte de contextes et d'enjeux sociaux, les révélateurs principaux des schémas d'attente qui sous-tendent l'interprétation des discours argumentatifs sont d'abord les identités de rôles endossées par les différents interlocuteurs (Fortin, 2006).

En ce sens, l'identité doit être envisagée en tant que produit social : comme l'ont montré Ghiglione et Trognon (Ghiglione et al., 1993), les interlocuteurs s'inscrivent au sein d'un espace co-géré en fonction d'un certain enjeu communicationnel ; l'identité des sujets ne pouvant se comprendre qu'en relation avec d'autres identités dans un espace de dialogue, en relation donc avec la reconnaissance communicationnelle dont elle fait l'objet. Les identités ne sont ainsi plus conçues comme isolées et uniques, mais comme le résultat de l'activité conjointe, et évolutive, de sujets en contact. Aussi, l'essentiel des productions discursives, dans les débats constituant notre corpus, réside dans la volonté de construire/proposer une image de soi, et des autres, qui puissent servir les objectifs argumentatifs :

Laurent Ruquier : je vais essayer de remettre un peu de bonne humeur sur ce plateau...

Mickaël Youn : il va falloir retirer 2 personnes alors [...]

Eric Zemmour : c'est vrai que nous on n'est pas des professionnels de la bonne humeur pour enfants de 4 ans

Mickaël Youn : j'ai pas bien entendu, c'est quoi le but de ce genre de joute verbale, c'est de te faire mousser, qu'est-ce que tu cherches?

Eric Zemmour : rien je réponds à tes questions c'est tout

\section{Le conflit : une interaction qui fait sens}

L'activité principale des interactants (y compris de l'animateur, Laurent Ruquier) est de se positionner par rapport aux autres en proposant ou en contestant - c'est-à-dire en négociant-des éléments de leur identité : 
Laurent Ruquier : je suis bien obligé de défendre mon bout de gras moi aussi

Mickaël Youn : pas de problème ce sont vos collègues de travail...

Laurent Ruquier : c'est pas que ça, c'est aussi moi, mon travail, c'est mon boulot, c'est mon job, mon émission

La capacité propre à se présenter, à dire qui je suis - et dans un même temps peut-être à médire l'autre - intervient donc pour la réalisation d'un « soi », dans la confirmation et/ou infirmation d'un profil identitaire. Le langage, ainsi considéré dans sa dimension de pratique sociale, possède donc une double fonction pour l'identité : une fonction à la fois instrumentale et objectivante permettant à chaque sujet, tour à tour, de se produire comme locuteur, et une fonction constitutive et subjectivante permettant à chaque locuteur de se spécifier, de se (re) définir, dans son accomplissement de locuteur, comme un être unique.

C'est la raison pour laquelle certaines séquences sont inaudibles si l'on ne prend pas la mesure de cet enjeu ${ }^{1}$ :

Mickaël Youn : c'est pas vrai...

Eric Zemmour : c'est pas vrai...

Mickaël Youn : bah moi je dis que tu te trompe

Eric Zemmour : c'est pas vrai...

Mickaël Youn : c'est pas vrai...

Eric Zemmour : Et bah c'est pas vrai...

Mickaël Youn : c'est pas vrai...

Eric Zemmour : et bah c'est ce que vous dites qui n'est pas vrai

Mickaël Youn : non c'est pas vrai

Les débattants doivent d'une part légitimer et crédibiliser leur activité de discours par une «bonne " représentation d'eux-mêmes et d'autre part s'évertuer à construire une représentation de l'autre qui soit incompatible avec son comportement de locuteur.

Eric Naulleau : j'adorerai me mettre sur la gueule avec lui, j'adorerai l'éparpiller façon puzzle sous les yeux équarquillés de la France qui se couche tard...

Nicolas Bedos : bah vas-y alors, vas-y fais-le...

1 Il s'agit ici d'avoir « le dernier mot », pour ne pas « perdre la face ». 
Eric Naulleau : mais tu es tellement fragile, tellement vulnérable à la critique que ça ne m'intéresse même pas parce que je sais que ce qui va se passer sur ce plateau va être recyclé dans un règlement de compte plus tard, comme tu le fais parce que moi au moins...

Nicolas Bedos : je fais ça...

Eric Naulleau : oui et vais en donner deux trois exemples...

Nicolas Bedos : Ah bah tu le fais finalement, le mec y dit je vais pas faire ça mais je vais le faire quand même [...]

Il s'agit donc, on le voit ici, pour chacun d'entre-eux « d'invalider la réussite énonciative d'un locuteur, laquelle vise la réalisation d'une représentation identitaire » (Burger, 1994, 265).

Francis Lalanne : un, tu ne sais pas ce qu'est un vers...

Eric Naulleau : oh bah alors ça...

Francis Lalanne : deux, tu ne sais pas c'est qu'est un rythme, trois tu ne sais pas ce qu'est une rime...

Eric Naulleau : (rire)

Francis Lalanne : tu crois que, tu crois que...

Eric Naulleau : c'est pas dans ton bouquin que je l'aurais appris $[\ldots]$

Francis Lalanne : pour qui tu te prends ? POUR QUI TU TE PRENDS ?

Mettre ainsi l'accent, comme le fait ici Eric Naulleau, sur l'incompétence propre à l'interlocuteur à dire qui il est (ce qui fonctionne par ailleurs tacitement comme une sorte de pré-requis au dialogue) revient à nier la possibilité même du débat ; cette stratégie argumentative implique ainsi une re-négociation du cadre relationnel construit par l'interlocuteur, mais au détriment de ce dernier. On retrouve dans l'exemple qui suit le même processus « argumentatif » :

Natacha Polony : ya chez vous ce besoin en permanence de sortir de son métier de journaliste, d'animateur, etc. pour la mise en scène de vous-même, je comprends pas...

Christophe Hondelatte : c'est quand même pas vous, qui faites le spectacle de vous-même tous les samedis soir à la télévision qu'allez me donner des leçons sur la manière de se mettre en scène... 
Natacha Polony : non moi je débats d'idées [...]

Natacha Polony : votre façon de mettre en scène, vous donnez l'impression d'envier les artistes [...] vous êtes un exemple de narcissisme contemporain $[\ldots]$

Christophe Hondelatte : du haut de votre grande réputation, de votre immense notoriété, de votre grandissime légitimité, vous venez me dire quelque chose pour laquelle vous n'êtes pas capable...

Natacha Polony : moi je ne me mets pas en scène comme vous... Christophe Hondelatte (à Laurent Ruquier) : c'est bon vous l'avez votre clash

Les " arguments » fonctionnent ici comme autant de tentatives (contestées ou non) de présentations de soi et de l'autre à l'intérieur d'un cadre et d'un enjeu eux-mêmes à négocier. C'est pourquoi la relation interpersonnelle se construit alors à deux niveaux structurels distincts : à la fois monologique et dialogique. La dimension polémique relève ainsi directement des habitudes relationnelles unissant les débattants, dans la mesure où les profils identitaires fonctionnent comme des gages de la reconnaissance illocutoire garantissant la réussite, ou non, de l'énonciation. Par exemple, dans l'extrait suivant, Eric Zemmour décide de reconnaître les questions formulées par Augustin Legrand comme construction d'un rapport d'autorité (maître-élève) qu'il récuse - ce qui lui permet à la fois de ne pas répondre à la question posée tout en dénonçant l'arrogance supposée de son interlocuteur :

Augustin Legrand: vous savez combien ya de mal logés monsieur Zemmour? en France

Eric Zemmour : bah dites moi, puisque vous savez tout Augustin Legrand : je vous pose la question vous répondez... Eric Zemmour : bah je suis pas, vous n'êtes pas mon professeur et je suis pas votre élève, on se calme là

\section{Conclusion : la voie d'une sociolinguistique de l'Action}

Les débats issus de l'émission On n'est pas couché, en plaçant les pratiques dialogiques dans la perspective de la confrontation, de l'affrontement verbal, inscrivent les stratégies argumentatives dans le cadre d'une structure duelle à fort encadrement rituel. Aussi, les propriétés situationnelles de la relation situent les débattants les uns 
par rapport aux autres : ils sont déjà dotés d'une histoire, d'un « style » interactif et d'une relation propre. Leurs échanges sont donc générateurs d'attentes à la fois comportementales et discursives et leur caractère répétitif les inscrits dans un cadre relationnel pré-établi.

En conséquence, les profils identitaires respectifs fonctionnent, a priori, comme une sorte d'horizon d'attente - au sens gricéen motivant des stratégies discursives, et façonnant également, et en même temps, les dispositions des (télé)spectateurs. Le débat permet donc aux invités de (re)construire des conventions interactionnelles et de les rendre manifestes. Il constitue ainsi ce lieu d'ajustement relationnel à l'intérieur duquel les accomplissements discursifs se négocient sur la base de critères identitaires; et où le véritable enjeu de la confrontation réside alors dans la volonté, réciproque, de valider la cohérence d'une certaine image de soi. Les interlocuteurs n'interagissent donc pas l'un sur l'autre, mais surtout l'un pour l'autre : les interactions conflictuelles n'ont de sens que dans un enlacement mutuel aux contextes et aux mondes, dans la mesure où il n'y a pas qu'un seul monde (naturel, et déjà constitué : paradigme platonicien), mais des mondes, des réalités à inventer et à négocier, tout un univers référentiel à co-construire - y compris dans le conflit - pour le rendre comm-un : « modelage mutuel d'un monde commun au moyen d'une action conjuguée : c'est notre réalisation sociale, par l'acte de langage, qui prête vie à notre monde » (Varela, 1990, 115).

Il s'agit donc de rompre avec une linguistique structurale interne, relevant du paradigme platonicien, pour s'engager, dans le sillon épistémologique tracé par l'interactionnisme symbolique, sur la voie d'une sociolinguistique de l'Action (Fortin, 2013), qui envisage le langage comme l'acte politique d'un vouloir vivre ensemble. Si le langage ne dit pas le monde (posture gnoséologique), il crée du sens, ayant vocation non plus à communiquer ou à transmettre de l'information (métaphore du conduit de la linguistique informationnelle) sur un monde déjà constitué, mais à concrétiser un monde en devenir.

\section{Références}

Amossy, R. Apologie de la polémique, PUF, Paris, 2014.

Amossy, R. (dir.), Images de soi dans le discours. La construction de l'ethos, Delachaux et Niestlé, Lausanne, 1999.

Amossy, R. La présentation de soi. Ethos et identité verbale, PUF, Paris, 2010. 
Burger, M. (1994). (Dé)construction de l'identité dans l'interaction verbale : aspects de la réussite énonciative de l'identité. Cahiers de linguistique française, Des mots et des discours : études contrastives et perspectives, (15).

Burger, M. (1995). L'identité négociée : " rapports de place(s) 》 dans un entretien télédiffusé. Cahiers de linguistique française, (17), Les différents plans d'organisation du dialogue et leurs interrelations, Actes du VIème colloque de pragmatique de Genève, $2^{\text {ème }}$ partie.

Charaudeau, P. «Contrats de communication et ritualisation dans les débats télévisés », in P. Charaudeau (Ed.), La Télévision, les débats culturels, « Apostrophes », coll. Langages, discours et sociétés, Didier Erudition, Paris, 1991.

Charaudeau, P. Le contrat médiatique, in les Dossiers audiovisuels, Ina, Paris, 2002, consultéle4juillet2016surlesitedePatrickCharaudeau-Livres, articles,publications. URL: http://www.patrick-charaudeau.com/Le-contrat-mediatique.html

Charaudeau, P., \& Ghiglione, R. (1997). La parole confisquée. Un genre télévisuel : le talk show, Dunod, Paris.

Corcuff, Ph. (1989). La sociologie de Bourdieu. 46. Le jeu politique, Editions Le Mascaret, Bordeaux.

Declercq, G. et al. (2003), La parole polémique, Honoré Champion, Paris.

Doury, M. (1997), Le débat immobile. L'argumentation dans le débat médiatique sur les parasciences, Paris, Editions Kimé.

Doury, M. \& Marcoccia, M. (1998). Construction des camps et circulation des slogans dans une interaction médiatique à thème politique : " Demain les Jeunes ». Actes du $1^{\text {er }}$ Symposium International sur l'analyse du discours, Madrid, (à paraître sur CDROM). [Cette étude s'inscrit dans un programme de recherche collectif au sein du Groupe de Recherches sur les Interactions Communicatives (CNRS - Université Lumière - Lyon 2).]

Ducrot, O. (1980). Les mots du discours, Minuit, Paris.

Flahault, F. (1978). La parole intermédiaire, Seuil, Paris.

Fortin, G. (2015), Pour une (socio)linguistique de l'Action : le langage comme instrument de l'invention/négociation du monde, in French Journal for Media Research [en ligne], Full text/Numéros en texte intégral, 4/2015 Les médias en Afrique du Sud Varia, Varia, mis à jour le : 27/06/2015, URL : http://frenchjournalformediaresearch. com/lodel/index.php?id=512.

Fortin, G. (2013), Une sociolinguistique de l'Action: construction du sens et interprétation en contexte, Sens et représentation en conflit, in Conceptualisation, signification et construction discursive, (sous la direction de Bellachhab, Abdelhadi \& Marie, Virginie), Collection: GRAMM-R. Etudes de linguistique française / GRAMM-R. Studies of French Linguistics, volume 12, Bruxelles, Bern, Berlin, Frankfurt am Main, New York, Oxford, Wien, 233 p

Fortin, G. (2007). L'approche socio-pragmatique en sciences du langage : principaux cadres conceptuels et perspectives. COMMposite, (1), pp. 109-129.

Fortin, G. (2006). Une dérive néo-sophistique ? Les pratiques argumentatives dans les débats politiques télévisés. Communication et Langages, (148), 53-67.

Ghiglione, R. et al., L'Homme communiquant, (1986), Colin, Paris.

Ghiglione, R., \& Trognon, A. (1993). Où va la pragmatique? De la pragmatique à la psychologie sociale. Grenoble : Presses de l'Université de Grenoble.

Goffman, E. (1973). La mise en scène de la vie quotidienne. La présentation de soi, T. 1. Le sens commun, Les Editions de Minuit, Paris. 
Goffman, E. (1976). Les rites d'interaction. Minuit, Paris.

Gumperz, J. (1989). Engager la conversation, introduction à la sociolinguistique interactionnelle, Minuit, Paris.

Habermas, J. (1987). Première considération intermédiaire : agir social, activité finalisé et communication. Théorie de l'agir communicationnel, tome 1 : Rationalité de l'agir et rationalisation de la société, Fayard, Paris, 283-345.

Mucchielli, A. (1983). L'analyse phénoménologique et structurale en sciences humaines, PUF, Paris.

Nel, N. (1990), Le débat télévisé, Armand Colin, Paris.

Nel, N. (1983). Le débat télévisé : méthodologie et Pédagogie. Pratiques, (37).

Plantin, Ch. (1990). Essais sur l'argumentation. Introduction linguistique à l'étude de la parole argumentative, Kimé, Paris.

Rouquette, S. (2002), Vie et mort des débats télévisés, Paris/Louvain-la-Neuve, De Boeck.

Varela, F. J. (1990). Connaître les sciences cognitives, tendances et perspectives. Paris : Le Seuil.

Windisch, U. (1986), Le K. O. verbal, la communication conflictuelle, Lausanne, L'Age d'Homme.

Winkin, Y. (2001). Anthropologie de la communication. De la théorie au terrain, Seuil, Paris. 